by ILAR subtype alone. It also reveals that the previously validated associations at position 13 of HLA-DRB1 are also correlated with JIAU in the polygo subtype suggesting that genetic risk factors will help refine risk within clinical subtypes. Conditioning on DRB1 alleles reveals that the secondary independent DRB1 position 69 association is also strongly associated in the polygo subset of this cohort. Together these results highlight the potential future use of genetics risk factors for risk classification for uveitis in patients with JIA

Disclosure of Interests: None declared

DOI: 10.1136/annrheumdis-2021-eular.430

\section{OP0015 GENOMIC RISK SCORE FOR ADVANCED OSTEOARTHRITIS IN OLDER ADULTS}

P. Lacaze ${ }^{1}$, G. Polekhina ${ }^{1}$, A. Bakshi ${ }^{1}$, M. Riaz ${ }^{1}$, A. Owen ${ }^{1}$, A. Franks ${ }^{1}$, Y. Wang ${ }^{1}$, J. Abidi ${ }^{2}$, J. Tiller ${ }^{1}$, S. Orchard ${ }^{1}$, J. Mcneil ${ }^{1}$, F. Cicuttini ${ }^{1}{ }^{1}$ Monash University, School of Public Health and Preventive Medicine, Melbourne, Australia; ${ }^{2}$ Alfred Hospital, Department of Medicine, Melbourne, Australia

Background: Osteoarthritis $(\mathrm{OA})$ is a major cause of disability, with treatment options mostly limited to advanced disease when joint replacement is indicated. Recent genome-wide association analysis more than doubled the number of OA-associated variants identified $(1,2)$, extending the feasibility of identification of high-risk individuals.

Objectives: Including these variants, we calculated polygenic risk scores (PRSs) and performed validation in a well-characterised population of older individuals. Methods: We calculated PRSs for knee and hip OA respectively, using joint replacement surgeries as markers of advanced disease in 12,724 older individuals of European descent in the ASPREE trial. We considered in-trial joint replacement (hospitalizations during median 4.7 years follow-up) and pre-trial joint replacement from self-reported medical history. Multivariable models examined the effect of PRS as a continuous variable (per standard deviation [SD]) and categorical variable (low-risk [0-20\%], medium-risk [21-80\%], high-risk [81-100\%] groups), adjusting for age, sex and BMI.

Results: Mean population age at baseline was 75 years and $54.9 \%$ of participants were female. In total, $1478(11.6 \%)$ participants had knee replacements and $1324(10.4 \%)$ had hip replacements. Female sex, higher BMI and age were associated with higher risk of knee and hip replacements. PRSs as continuous variables per SD were associated with knee (odds ratio [OR] 1.11, 95\% confidence interval $[\mathrm{Cl}] 1.04-1.17)$ and hip (OR 1.18, 95\% $\mathrm{Cl} 1.11-1.25)$ replacements. We found meaningfully different rates of knee or hip joint replacement occurring between low-, medium- and high-risk PRS groups. Participants in the high-risk PRS group, compared with the low, had a higher risk of knee replacements $(\mathrm{OR}=1.35, \mathrm{Cl} 1.12-1.62)$, and hip replacements (OR 1.66, 95\% Cl 1.37-2.02). We found no interaction between PRS and sex, and no collinearity between PRS and BMI, suggesting PRS is an independent risk factor for OA.

Conclusion: Joint-specific genomic risk scores predict advanced OA in older adults, independent of age, sex and BMI. Stronger associations are observed for hip versus knee OA. Our study provides some of the first evidence of potential clinical utility of genomic risk prediction for $\mathrm{OA}$, which may help identify individuals who would benefit most from targeted clinical management and preventive intervention. REFERENCES:

[1] Tachmazidou I, Hatzikotoulas K, Southam L, et al. 2019. Identification of new therapeutic targets for osteoarthritis through genome-wide analyses of UK Biobank data. Nat Genet, 51, 230-236

[2] Zengini E, Hatzikotoulas K, Tachmazidou I, et al. 2018. Genome-wide analyses using UK Biobank data provide insights into the genetic architecture of osteoarthritis. Nat Genet, 50, 549-558

Disclosure of Interests: None declared

DOI: 10.1136/annrheumdis-2021-eular.2918

\section{OP0016 IDENTIFICATION OF FUNCTIONAL VARIANTS IN THE RHEUMATOID ARTHRITIS ASSOCIATED JAZF1 LOCUS IN SYNOVIAL FIBROBLASTS}

M. Houtman ${ }^{1}$, X. Ge ${ }^{2}$, A. Mcgovern ${ }^{2}$, K. Klein ${ }^{1}$, G. Orozco ${ }^{2}$, M. Frank Bertoncelj ${ }^{1}$, M. Marks ${ }^{3}$, O. Distler ${ }^{1}$, P. Martin ${ }^{2}$, S. Eyre ${ }^{2}$, C. Ospelt ${ }^{1} .{ }^{1}$ University Hospital Zurich, University of Zurich, Center of Experimental Rheumatology, Department of Rheumatology, Zurich, Switzerland; ${ }^{2}$ The University of Manchester, Versus Arthritis Centre for Genetics and Genomics, School of Biological Sciences, Faculty of Biology, Medicine and Health, Manchester, United Kingdom; ${ }^{3}$ Schulthess Klinik, Department of Teaching, Research and Development, Zurich, Switzerland

Background: Over the past decade, genome wide association studies (GWAS) have identified the JAZF1 locus as a risk locus for several autoimmune diseases, including rheumatoid arthritis (RA $)^{1}$. However, the exact causal variants in the JAZF1 locus and their underlying regulatory events contributing to RA are still not known. Here, we focus on the effect of these variants on gene expression in synovial fibroblasts (SF).

Objectives: To characterize the functional consequences of RA-causal variants in the JAZF1 locus in SF.

Methods: Genetic fine-mapping of RA loci was conducted by computing sets of credible variants driving GWAS signals. These credible variant sets were integrated with DNA architecture (ChIP-seq), 3D chromatin interactions (3C, HiC and capture $\mathrm{HiC}$ ), DNA accessibility (ATAC-seq) and gene expression (RNA-seq and CAGE-seq) datasets to select putative RA-causal variants in SF. Selected variants in the JAZF1 locus were tested for regulatory function by luciferase reporter assays and electrophoretic mobility shift assays (EMSA) in the fibrosarcoma cell line HT1080. The JASPAR2020 database was used to identify putative transcription factors (TF) binding to the selected variants. The expression of HOTTIP was measured by quantitative PCR in hand SF $(n=23)$. Genotyping was done by pyrosequencing.

Results: Genetic fine mapping revealed 47 variants in the JAZF1 locus. Integration of these variants with the chromatin datasets prioritized rs 2158624 rs57585717 and rs 186735625 as the top candidates (posterior probability of causality $>0.1$ ) in the JAZF1 locus. We found that rs2158624 and rs186735625 are located in the vicinity of enhancer elements in SF as determined by ATAC-seq. In addition, the region of rs2158624 exhibited strong chromatin interactions with the genomic region of HOTTIP and HOXA13. Both these transcripts were previously shown to be specifically expressed in SF isolated from hands and feet ${ }^{2}$. Based on this, we selected rs2158624 as the most promising candidate in the JAZF1 locus. We found that the rs2158624-C allele (risk) is associated with lower expression of HOTTIP, but not HOXA13, in hand SF compared to the rs2158624-T allele (non-risk) $(p=0.02)$. Luciferase assays in HT1080 cells demonstrated enhancer activity with both the rs2158624-C allele $(p=0.006)$ and T allele $(p=0.04)$, with no significant difference in enhancer activity between the rs2158624-C and $T$ allele. EMSAs identified stronger specific binding of HT1080-cell nuclear extract for the rs2158624-T allele than for the $C$ allele (risk). Based on the JASPAR2020 database, we identified NFAT5 as a potential TF that can bind to rs2158624 and may regulate the expression of HOTTIP.

Conclusion: We were able to substantially narrow down the potential functional variants in the JAZF1 locus using our data integration approach and functional assays. We suggest that the risk allele of rs2158624 influences the binding of TFs controlling the expression of the long non-coding RNA HOTTIP in SF, which might confer specific risk to develop RA in hands.

\section{REFERENCES:}

[1] Okada Y et al. Genetic of rheumatoid arthritis contributes to biology and drug discovery. Nature 2014;506:376.

[2] Frank-Bertoncelj $\mathrm{M}$ et al. Epigenetically-driven anatomical diversity of synovial fibroblasts guides joint-specific fibroblast functions. Nat Commun 2017;8:14852.

Disclosure of Interests: Miranda Houtman: None declared, Xiangyu Ge: None declared, Amanda McGovern: None declared, Kerstin Klein: None declared, Gisela Orozco: None declared, Mojca Frank Bertoncelj: None declared, Miriam Marks: None declared, Oliver Distler Speakers bureau: Bayer, Boehringer Ingelheim, iQone, Medscape, MSD, Novartis, Pfizer and Roche, Consultant of: Abbvie Acceleron Pharma, Amgen, AnaMar, Arxx Therapeutics, Bayer, Baecon Discovery, Boehringer, CSL Behring, ChemomAb, Corbus Pharmaceuticals, Galapagos NV, GSK, Glenmark Pharmaceuticals, Horizon Pharmaceuticals, Inventiva Italfarmaco, iQvia, Kymera, Lilly, Medac, Medscape, Mitsubishi Tanabe Pharma, MSD, Pfizer, Roche, Roivant Sciences, Sanofi and UCB, Grant/research suppor from: Kymera Therapeutics and Mitsubishi Tanabe, Paul Martin: None declared, Stephen Eyre: None declared, Caroline Ospelt: None declared DOI: 10.1136/annrheumdis-2021-eular.1400

\section{OP0017 \\ VALIDATION OF GWAS-IDENTIFIED VARIANTS FOR ANTI-TNF DRUG RESPONSE IN RHEUMATOID ARTHRITIS: A META-ANALYSIS OF THREE LARGE COHORTS}

J. M. Sánchez-Maldonado ${ }^{1}$, R. Cáliz Cáliz² ${ }^{2}$ M. Á. López-Nevot ${ }^{3}$, A. Moñiz-

Díez $^{1}$, A. J. Cabrera-Serrano ${ }^{1}$, H. Canhão ${ }^{4}$, R. Ter Horst ${ }^{5}$, A. Escudero Contreras $^{6}$, S. Sorensen ${ }^{7}$, M. L. Hetland ${ }^{8}$, M. Á. Ferrer González ${ }^{2}$, B. Glintborg ${ }^{8,9}$, I. Filipescu ${ }^{10}$, E. Perez-Pampín ${ }^{11}$, P. Conesa-Zamora ${ }^{12}$, J. Swierkot ${ }^{13}$, A. Den Broeder ${ }^{14}$, S. De Vita ${ }^{15}$, S. E. Hove Jaconsen ${ }^{16}$, L. Quartuccio ${ }^{15}$, Y. LI ${ }^{5}$, M. Netea ${ }^{5}$, M. J. H. Coenen ${ }^{14}$, V. Andersen ${ }^{8,9}$ J. E. Fonseca ${ }^{17}$, K. Bogunia-Kubik ${ }^{18}$, E. Collantes Estevez ${ }^{6}$, J. Sainz ${ }^{1,19}$ on behalf of REPAIR consortium. ${ }^{1}$ GENYO. Center for Genomics and Oncological Research, Genomic Oncology, Granada, Spain; ${ }^{2}$ Virgen de las Nieves University Hospital, Rheumatology Deparment, Granada, Spain; ${ }^{3}$ Virgen de las Nieves University Hospital, Immunology Department, Granada, Spain; ${ }^{4}$ Universidade Nova de Lisboa, CEDOC, EpiDoC Unit, NOVA Medical School and National School of Public Health, Lisboa, Portugal; ${ }^{5}$ Radboud University 